\title{
Factors influencing adolescent wholegrain intake
}

\author{
M. Kamar ${ }^{1}$, S. Albar ${ }^{1}$, S. Hugh-Jones ${ }^{2}$ and C. E. L. Evans ${ }^{1}$ \\ ${ }^{1}$ Nutritional Epidemiology Group, School of Food Science and Nutrition, University of Leeds, LS2 9JT and ${ }^{2}$ Institue of \\ Psychological Sciences, Faculty of Medicine and Health, University of Leeds, LS2 9JT
}

Wholegrain consumption has been linked to reduced risks of hypertension, diabetes, obesity, cardiovascular disease, and various types of cancer ${ }^{(1,2)}$. Almost a third of UK adults and children do not consume wholegrain and as little as $5 \%$ reach the recommended three portions per day ${ }^{(3,4)}$. Little has been done to explore wholegrain consumption correlates, and factors influencing wholegrain intake in UK adolescents are unknown. This qualitative study explored the socio-economic, environmental, lifestyle, and psychological factors influencing adolescent wholegrain intake.

Participants were male and female adolescents $(n=50)$ aged $11-16$ years from mixed socioeconomic backgrounds and ethnicities, recruited through schools in the Leeds area. Five focus groups were conducted which included wholegrain identification games and questions based on the theory of planned behaviour, which explored attitudes, knowledge, consumption, intentions to consume wholegrain and barriers to consumption. Focus groups were audio-recorded and analysed using template analysis ${ }^{(5)}$.

The majority $(86 \%)$ of the participants had tried wholegrain products in the past. Cereal products were the most popular, followed by bread and oat-based products, respectively. Fibre content, digestive health and slow energy release were the most recognised wholegrain health benefits, whereas the least mentioned were the cancer-fighting anti-oxidant properties of wholegrain. Expressions of dislike for wholegrain taste were slightly higher than those of approval for most products. In the identification game, participants were able to correctly identify slightly less than half of the wholegrain products. The predominant barriers to wholegrain intake were reported to be sensory properties and taste, followed by lack of awareness of health benefits and availability in shops and schools. Suggested facilitators of consumption were advertisements and educational campaigns, followed by improved sensory appeal, increased availability, varieties, and tailoring of products for young people.

This study identified barriers and facilitators to wholegrain consumption in UK adolescents. This research will considerably add to the limited existing knowledge of promotion of wholegrain food consumption to improve health in this target group. More research should address wholegrain intake correlates and interventions to improve WG awareness, availability, and consumption.

1. Seal C. J. \& Brownlee I. A. (2010) Cereal Chem. 87, 167-174.

2. Smith A. T. Kuznesof S. Richardson D. P. et al. (2003) Proc Nutr Soc 62, 455-467.

3. Lang R., Thane C. W., Bolton-Smith C. et al. (2003) Public Health Nutr 6, 479-484.

4. The HEALTHGRAIN Project (2010) FOOD-CT-2005-514008.

5. KING N., Symon G., Cassell C. (1998) Template analysis. Sage, Thousand Oaks, CA 118-134 